\title{
Practice guidelines for the renal biopsy
}

\author{
Patrick D Walker ${ }^{1}$, Tito Cavallo², Stephen M Bonsib ${ }^{3}$, The Ad Hoc Committee on Renal \\ Biopsy Guidelines of the Renal Pathology Society
${ }^{1}$ Nephropathology Associates, Little Rock, AR, USA; ${ }^{2}$ Department of Pathology and Laboratory Medicine, University of Cincinnati College of Medicine, Cincinnati OH, USA and ${ }^{3}$ Department of Pathology and
Laboratory Medicine, Indiana University School of Medicine, Indianapolis, IN, USA

\begin{abstract}
Biopsy of the kidney should never be undertaken without careful consideration of the risks vs benefits. Given the importance of a correct diagnosis in the treatment and prognosis of renal disease, the pathological evaluation should use all available modalities. Native kidney biopsies require examination by light microscopy, immunohistochemistry and electron microscopy. The processing of the renal biopsy is complex and requires the support of a fully equipped anatomic pathology laboratory. Technical expertise is required to process the small fragments of tissue and to produce sections of highest quality. The correct diagnosis requires a welltrained renal pathologist with thorough knowledge of not only renal pathology but also renal medicine in order to correlate intricate tissue-derived information with detailed clinical data. In view of the importance and consequences of the pathologic diagnosis, the Renal Pathology Society appointed an Ad Hoc Committee on Practice Guidelines, to define the essential ingredients necessary to provide quality renal pathology diagnoses. This document incorporates the consensus opinions of the committee and the RPS membership at large. Modern Pathology (2004) 17, 1555-1563, advance online publication, 23 July 2004; doi:10.1038/modpathol.3800239
\end{abstract}

Keywords: renal biopsy; glomerulonephritis; allograft; transplant rejection

The renal biopsy, though a relatively safe medical procedure, should be undertaken only after serious consideration of possible morbidity and rare mortality that can occur with this invasive technique. ${ }^{1}$ Owing to the nature of the diseases to be detected, the sample of renal tissue needs to be examined with optimal methods to allow for a complete evaluation, including light microscopy (LM), immunohistochemistry (IHC, includes either immunofluorescence (IF) or immunoperoxidase (IP)) and electron microscopy (EM). The correct diagnosis requires a well-trained renal pathologist with thorough knowledge of not only renal pathology but also renal medicine in order to correlate intricate tissuederived information with detailed and sometimes subtle clinical data in order to provide the best possible clinicopathologic diagnosis or diagnoses.

The Renal Pathology Society formed an Ad Hoc Committee on Renal Biopsy Guidelines to develop recommendations regarding the processing and evaluation of renal biopsy specimens. This was followed by an intensive several-year period of

Correspondence: Dr PD Walker, MD, Nephropathology Associates, 10802 Executive Center Drive, Suite 111, Little Rock, AR 72211, USA.

E-mail: walkerpd@kidneybx.com

Received 29 March 2004; revised 17 June 2004; accepted 24 June 2004; published online 23 July 2004 refinement. The guidelines would first be reviewed by the Executive Board of the Renal Pathology Society and subsequently submitted to the membership for discussion, debate and suggestions (see the contributor list in Acknowledgments). The committee then revised the guidelines and the process would begin anew. The techniques and guidelines appropriate for adequate evaluation of a renal biopsy and the recommended qualification standards for those interpreting renal biopsies presented in the following sections are the result of this rigorous process.

\section{Renal biopsy fixation and processing}

The renal biopsy should be accompanied by adequate clinical information to enable proper interpretation of findings. Many laboratories provide a special clinical information form to ease the recording of the overall renal syndrome, symptoms and laboratory data. Although this does not replace direct communication with the submitting physician, this basic medical information provides a good initial background for overall interpretation of the renal biopsy. The use of a dissecting microscope can be of assistance in assessing sample adequacy. Another alternative is the use of a standard light microscope. The tissue is placed on a glass slide 
with normal saline and examined with or without a coverslip producing a wet mount. ${ }^{2}$ A trained observer can recognize fat, skeletal muscle, and other nonrenal tissue. Knowledge of the glomerular content of the sample can guide division of tissue for the various histologic modalities (Figure 1).

In the absence of direct glomerular visualization, a standard protocol for dividing the tissue obtained at each 'pass' should be used to avoid inadequate glomerular sampling for LM, IF or EM
(Figure 2). There are several acceptable approaches; laboratories that perform IHC on fixed embedded tissue have even more options. The standard approach is to first procure tissue for EM from each core by removing $1 \mathrm{~mm}$ cubes from the ends and placing them in formalin, cooled glutaraldehyde or other fixative suitable for EM. Some clinicians prefer that the pathology laboratory obtain tissue for EM from the ends of the formalinfixed tissue.
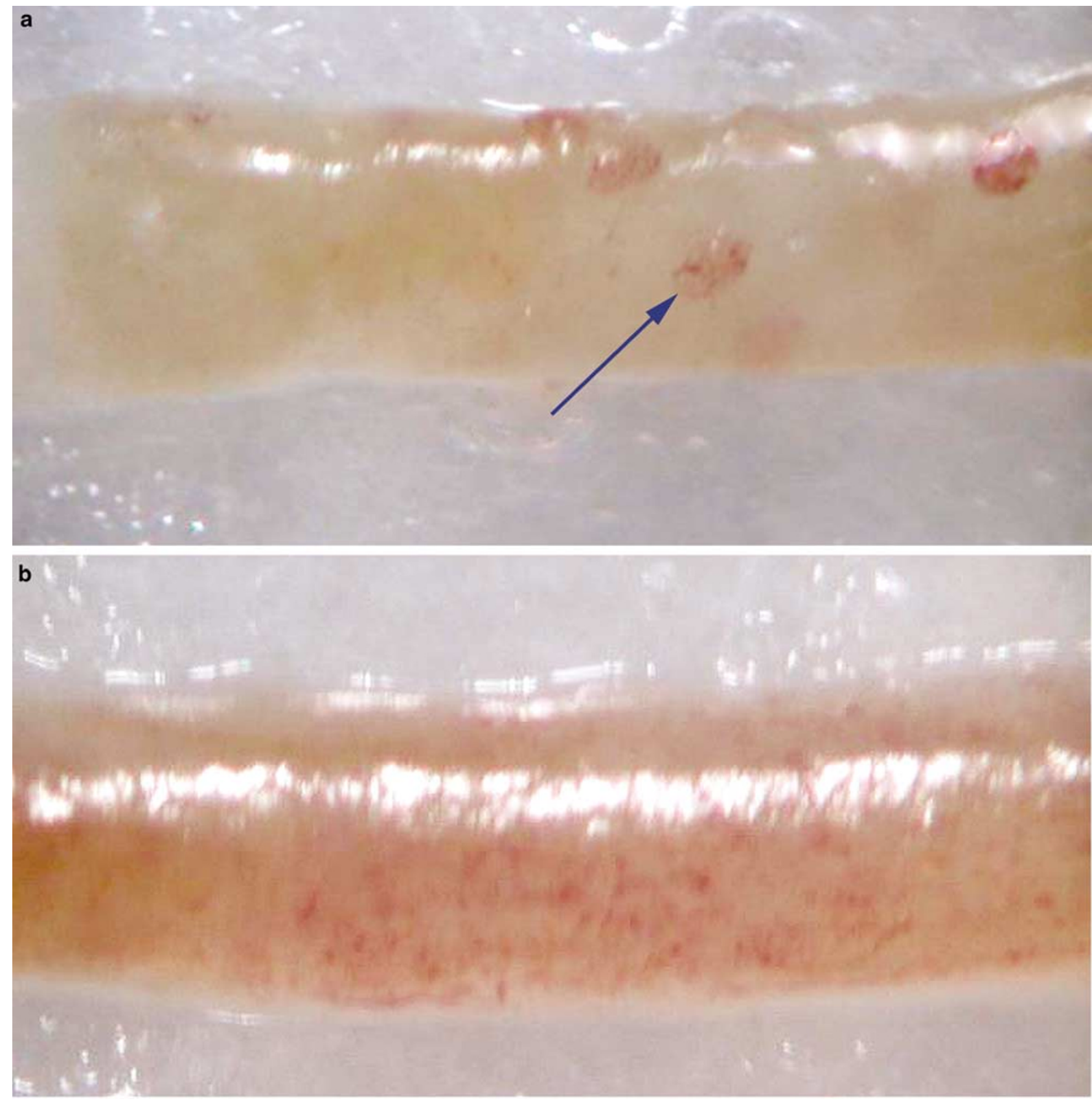

Figure 1 Renal biopsy specimen as seen with a dissecting microscope. (a) Renal cortex, note the glomeruli, recognized as round red areas (wet preparation $\times 10$ ). (b) Renal medulla, reddish vasculature is present but no glomeruli seen (wet preparation $\times 10$, photographs contributed by Alexis Harris, MD). 


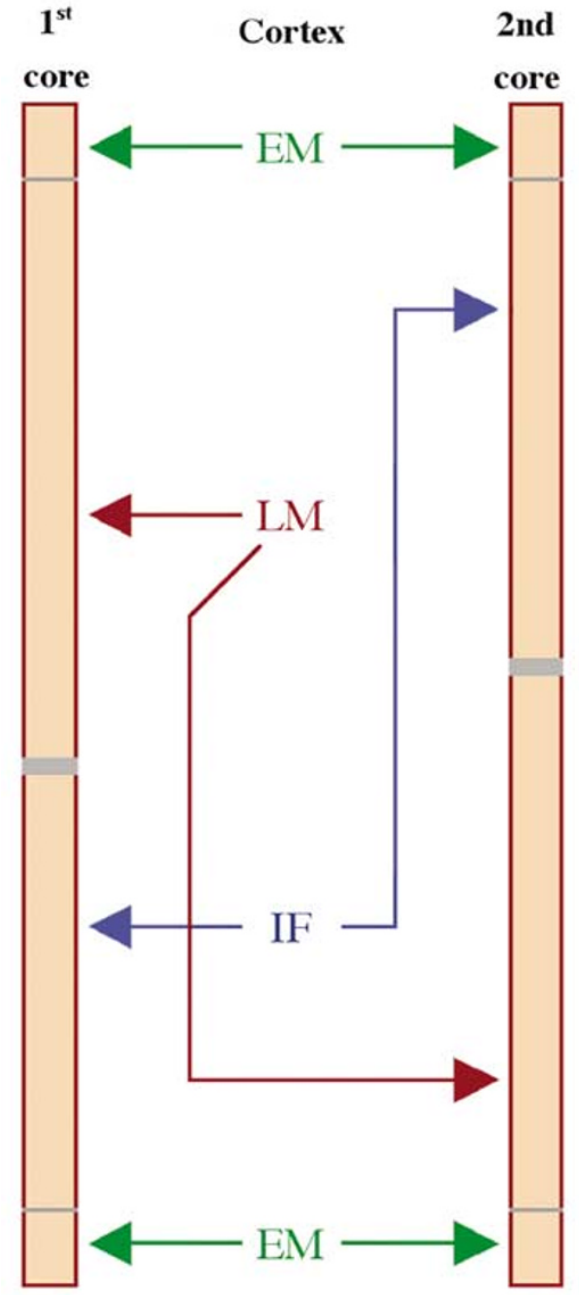

Medulla

Figure 2 Diagram to illustrate division of renal biopsy cores in the absence of a dissecting microscope for laboratories using IF. The ends from all cores are taken for EM with the remainder divided for LM and IF.

If the specimen is to be sent to a laboratory that uses IF, the first core can be cut in half by crosssectioning and the larger piece placed in formalin or another fixative suitable for LM; the smaller portion is saved for immunofluorescence evaluation. If a second core is obtained, the ends should be taken for $\mathrm{EM}$ and the specimen again divided almost in half with the larger tissue core now kept for IF and the smaller for LM. Alternatively, if both cores contain cortex, one core can used for LM and one core for IF. Tissue from further passes should be divided to balance whatever was obtained initially.

If tissue is limited, the clinical differential diagnosis may drive the division of material, for example, in cases of rapidly progressive glomerulonephritis, an attempt to secure sufficient material for IHC to rule out antiglomerular basement membrane antibodies is important. Although artefacts will be induced, frozen tissue used for IF can be subsequently processed for either LM or EM and, depending upon the questions to be addressed, useful information can still be obtained.

The biopsy specimen must be handled gently when removed from the biopsy needle; an $18 \mathrm{G}$ needle or a thin wooden stick, for example, a toothpick, is ideal. Normal saline can be also used to wash the sample off the needle. To avoid crush artefact, forceps should not be used. Pulling and stretching the tissue during removal from the needle must also be avoided. Desiccation artefacts and osmotic injury may result from placing the tissue on dry gauze or gauze soaked with water, respectively. Other sources of artefact include freezing the entire sample, or placing the sample in ice-cold saline. If the biopsy cores are sent to the pathology laboratory for division and processing, they may be placed in transport fluid (usually tissue culture medium), physiologic saline-moistened gauze or filter paper carefully folded over the tissue.

The sample should be cut with a fresh scalpel or single-edged blade (one that has been cleansed of oil and not been exposed to fixative). The portion for IF is gently picked up with a separate clean tool and placed in transport solution (some centers use tissue culture medium) remaining untouched by formalin or other fixatives. The remainder is placed promptly in fixative for LM and EM because good LM and EM morphology is dependent upon the rapidity of tissue fixation.

If the pathology laboratory uses IP instead of IF, biopsy handling is simplified. The material for IP is taken from the formalin-fixed material also used for LM requiring only that the ends of all pieces be saved for EM.

\section{LM}

\section{Fixation}

The most commonly used fixative for LM is buffered, $10 \%$ aqueous formaldehyde solution (formalin). Formalin is stable at room temperature, provides acceptable morphology, and allows IHC or molecular studies to be performed. Some laboratories prefer alcoholic Bouin's, ${ }^{3}$ Duboscq-Brasil ${ }^{4}$ or Zenker's fixatives that provide better preservation of certain morphologic details. However, these fixatives limit recovery of material for EM, IP or molecular studies, and require additional precautions and handling. Bouin's and Duboscq-Brasil fluid contain picric acid that is highly explosive when dry and can be a disposal problem. Some laboratories prefer $4 \%$ paraformaldehyde to optimize tissue suitability for LM, IHC and EM and for possible research applications including in situ hybridization (ISH) studies. Owing to a decrease in polymerization of critical components, this fixative provides superior molecular stabilization for IHC and ISH. It also provides preservation of 
morphologic details equivalent to formalin. ${ }^{5,6}$ Methacarn, a modified Carnoys fixative (methyl alcohol $60 \%$, chloroform $30 \%$, glacial acetic acid $10 \%$ ), provides good fixation for LM and EM and, in some studies, has provided excellent recovery of mRNA, ${ }^{7}$ though not in others. ${ }^{8}$

The material processed for LM can serve as reserve material for IHC or EM if either of these other modalities is found lacking glomeruli. Formalin fixation and paraffin embedding is not an impediment to detailed electron microscopic evaluation and rapid reprocessing is a relatively simple procedure. ${ }^{9-11}$

\section{Processing and sectioning}

To avoid specimen loss during processing, the specimen for LM should be gently wrapped in lens paper that has been prewetted with fixative. Neither sponges nor plastic embedding bags should be used because mechanical artefact almost always occurs. Tissues can be processed overnight using a protocol appropriate for small biopsy samples. Alternatively, rapid processing protocols are available, some employing a microwave fixation step, that can be used for same-day processing. ${ }^{12-14}$

\section{Sectioning and staining}

Serial sections of $2 \mu \mathrm{m}$ thickness are cut and at least two sections should be placed on each slide. There are many acceptable staining protocols; most include staining alternating slides with hematoxylin and eosin stain (H\&E) periodic acid-Schiff reaction (PAS), silver methenamine and trichrome stains.

\section{IHC}

\section{Preparation}

IF is best performed on unfixed, frozen sections. Tissues can be transported to the laboratory fresh on saline-soaked gauze or in Michel's fixative (Modified Michel's Tissue Fixative (Wampole Laboratories, Cranbury, NJ, USA) sometimes referred to as 'Zeus medium'). ${ }^{15}$ Serial sections are cut at $2-4 \mu \mathrm{m}$ in a cryostat. IP staining requires no special tissue preparation in that the same formalin-fixed, paraffin-embedded material used for LM is also used for IP.

\section{Staining}

The antigens that should be routinely examined include: immunoglobulins (primarily IgG, IgM and IgA), complement components (primarily C3, C1q, and C4), fibrin, and kappa and lambda light chains. Additional antibodies may be required in specific circumstances, for example, amyloid speciation, ${ }^{16,17}$ collagen IV alpha chains in hereditary nephritis, ${ }^{18,19}$ IgG subclasses, ${ }^{20,21}$ virus identification, ${ }^{22-25}$ lymphocyte phenotyping in allografts in suspected cases of PTLD, ${ }^{26,27}$ C4d in allograft biopsies, ${ }^{28,29}$ etc. In the absence of appropriate tissue in the IF sample, paraffin-embedded material can be examined using IP techniques (see later).

\section{Controls}

IHC controls should include a negative control (no antibody applied) and a positive control (albumin can serve this purpose) for each run. Internal controls exist for many of the antigens in routine use. For example, IgA is generally present in tubular casts, IgG in protein droplets and C3 in blood vessels. Each time a new vial of antibody is opened, the correct dilution should be determined using known positive slides.

\section{IF vs IP}

IP has almost completely replaced IF in routine surgical pathology and in many renal pathology laboratories. Well-developed antigen-retrieval procedures and the widespread availability of automated IP stainers provide reproducible results in kidney biopsies. Advantages of IP include correlation of the LM and IP since sections come from a single block, and an expensive IF microscope attachment is not needed. IP material is also a more permanent sample allowing repeated examination without fading.

Still, IP has certain drawbacks. Complement antigens require careful, time-consuming antigenretrieval procedures because of antigen masking during processing into paraffin. A false negative can be a concern. Interpretation of IP slides is also complicated by potentially higher background in renal tissues and the difficulty of titrating the final color product to avoid over- or understaining. Also, since the IP signal is a reaction product dependent on interaction of substrate and enzyme, diffusion artefacts can create problems in interpretation.

Immunofluorescence is the choice for many nephropathologists. The use of dark field microscopy for IF produces a very high signal-to-noise ratio. Accurate localization of deposits is possible in most laboratories with good frozen section technique, and excellent resolution is achievable with fluorescence microscopes having epifluorescence attachments. Overall, the choice between IF or IP is based on the experience and technical resources of the nephropathologist. Both methods can provide excellent material and, in fact, they can complement one another in various clinical settings.

\section{EM}

The tissue for EM may be fixed in $2-3 \%$ glutaraldehyde or $1-4 \%$ paraformaldehyde. Adequate fixation can also be obtained when tissue is fixed in buffered formalin. EM cannot be performed on tissues exposed to mercury-based fixatives (Zenker's, B-5, etc). The tissue sent in Michel's transport medium would have poor ultrastructural 
preservation since this is not a fixative solution. Rapid placement of the sample into fixative will provide the best outcome. Tissue can be reprocessed from the paraffin block, or the frozen block if no glomeruli are available in the EM sample. Although severe cellular artefacts may result when frozen and paraffin-embedded tissue are processed for EM, the GBM and immune deposits are usually sufficiently intact for evaluation.

Toluidine blue-stained, $1 \mu \mathrm{m}$ thick, so-called 'thick' sections, are examined to identify appropriate structures for thin sectioning and examination with the electron microscope. Thick sections are also useful to supplement the paraffin material (eg a lesion of focal segmental sclerosis may only be present on the thick section). In general, one or two glomeruli are examined ultrastructurally. Low-, medium- and high-magnification photographs are taken to include both capillary loops and mesangial areas. The tubulointerstitium and vessels are also examined and pertinent photographs taken to illustrate any abnormalities in these areas.

\section{Interpretation of the renal biopsy}

The evaluation of a kidney biopsy includes examination of multiple serial sections each with several tissue slices, stained with a variety of stains to be examined by LM and IHC. 'Thick sections' for EM are also reviewed for additional information. Careful evaluation of glomeruli, tubules, the interstitium and the vessels is required. The final report should provide a glomerular count with the number showing global and/or segmental sclerosis. In certain situations, other glomerular lesions should be counted (eg number of crescents, subtyped into cellular, fibrocellular and fibrous, etc). A description of alterations in tubules, interstitium and vessels should also be included. Each slide represents another level that may reveal additional findings, re-emphasizing the importance of meticulous examination of all materials. Rapid turnaround is required to obtain maximum utility from information derived from the renal biopsy. Two days for LM and IF and 3-5 days for EM should be considered routine. Finally, a renal biopsy should never be interpreted in a clinical vacuum. A nephropathologist must have a thorough understanding of renal disease as well as good communication with the nephrologists caring for the patients. This allows an accurate correlation of clinical information with the observed pathologic processes and this combination will lead to the correct final diagnosis.

\section{The Native Kidney Biopsy}

Native kidney biopsies provide maximum information when evaluated by LM, IHC and EM. ${ }^{30-32}$ Whether the use of all three modalities is necessary for all biopsies has been debated, and certainly some
Table 1 Renal biopsy diagnosis requires LM, IF and EM

Diagnoses overlooked without IHC

Light chain-associated diseases

AL amyloid

Monoclonal immunoglobulin deposition disease

Light chain cast nephropathy

IgA nephropathy/Henoch-Shonlein purpura

IgM nephropathy

C1q nephropathy

Antiglomerular basement membrane disease

Humoral (C4d) transplant rejection

Fibronectin glomerulopathy

Diagnoses overlooked without EM

Fibrillary glomerulopathy/immunotactoid glomerulopathy

Nail-patella syndrome

Lipoprotein glomerulopathy

Dense deposit disease

Alport's syndrome

Thin glomerular basement membrane nephropathy

Collagenofibrotic glomerulopathy

nephropathologists use their discretion in selected cases. ${ }^{33}$ Still, less than a complete workup should be a rare exception, usually resulting from an inadequate specimen for one of the studies, LM, IF or EM. Biopsy diagnoses generated without IHC or EM data may result in an erroneous or incomplete diagnosis (Table 1) because a number of entities can only be recognized by IHC or EM.

If one study is intentionally omitted it is usually EM. Recent reviews, however, have demonstrated the utility of EM in renal biopsy diagnosis and reconfirmed the necessity of routinely performing electron microscopic evaluation. ${ }^{32,34}$ Haas $^{34}$ showed that the usefulness of EM has not declined since the earlier reports documenting the need for EM published in the early 1960s and 1970s. While EM was absolutely necessary to make a correct diagnosis in $21 \%$ of cases, EM evaluation resulted in clinically relevant refinement of or addition to the diagnosis in another $24 \%$. Examples of the latter include findings of thin glomerular basement membranes (GBMs) to account for otherwise unexplained hematuria in a patient with interstitial nephritis or acute tubular necrosis, tubuloreticular inclusions in a reportedly HIV-negative patient with collapsing glomerulopathy, among others. Although in retrospect, as many as $50 \%$ of cases do not require EM, it is not possible in an individual biopsy to determine if it can be completed without EM until the EM findings are actually known.

\section{The Renal Transplant Biopsy}

\section{Donor biopsy}

Questions regarding the suitability of a cadaveric kidney for transplantation may arise, particularly with the increasing use of older donors. Intraoperative frozen sections are frequently employed in 
these situations. The information extractable from a frozen section is directly linked to the quality of the section generated. A frozen section of acceptable quality is adequate to assess the percentage of globally sclerotic glomeruli and the extent of arteriosclerosis to identify major glomerular lesions and significant interstitial inflammation. Attempts to diagnose glomerulonephritis, quantitate interstitial fibrosis or identify mild acute tubular necrosis are frequently beyond the capabilities of the usual frozen tissue section.

The easiest determination is quantitation of glomerulosclerosis and this is usually the main point of interest to the surgeon. Randhawa et $a l^{35}$ correlated donor biopsy histologic findings with 12-month post-transplant outcome. Using rigorous multivariate analysis, they demonstrated that with each increment in global sclerosis (graded 0, 1, 2, 3; $0,1-10,11-20,21-30 \%)$, the odds ratio for a worse outcome increased by $2.0(P<0.03)$ after correcting for recipient age. In a more recent study, Escofet et $a{ }^{36}$ have confirmed the importance of glomerulosclerosis as a predictor of outcome. Importantly, Wang et $a l^{37}$ have shown that for a statistically significant prediction of outcome based on glomerulosclerosis, at least 25 glomeruli are needed in the baseline biopsy. Although there is also a strong correlation between the presence of interstitial fibrosis and a worse outcome,$^{38}$ this determination is difficult to impossible on a frozen section of donor kidney. As with all frozen sections, the remaining material should be formalin-fixed and submitted for LM. When time is less critical, rapid processing after formalin fixation can provide information far superior to that available on frozen section.

\section{Transplant biopsies for allograft dysfunction}

In many centers, transplant biopsies are handled differently than native kidney biopsies. A minimum of two biopsy cores is indicated since the sensitivity of a single core for rejection is $91 \%$; the addition of the second core improves the sensitivity to about $99 \%{ }^{39}$ The same histologic staining protocol is usually followed. Often IF and EM are omitted unless there is clinical suspicion of recurrent disease. Recent appreciation of the correlation between antidonor HLA antibodies, ${ }^{28,38}$ and C4d deposition along peritubular capillary endothelium and resistant rejections mandating more aggressive therapeutic intervention, has led to the recommendation of all or most biopsies for acute dysfunction be stained for C4d. Both IF and IP procedures exist for this antigen.

The use of a reproducible classification scheme for rejection that provides significant prognostic information is important. The most widely accepted system is the Banff Classification. ${ }^{40,41}$ This is a working formulation that continues to evolve as evidenced by the recent addition of antibodymediated rejection criteria. ${ }^{42}$

\section{New directions}

'It is hard for the present day nephrologist or nephropathologist to conceive how renal pathology was understood prior to renal biopsy" ${ }^{\prime 3}$ (quoted in Pirani ${ }^{44}$ ). The renal biopsy was a significant underpinning for many of the great advances seen in the understanding of renal diseases during the last 50 years and continues to play an important role. Accurate diagnosis and classification of renal diseases has also led to the development of more specific and superior therapy. Still, the renal biopsy as evaluated now, cannot always suggest the best choice between various available therapeutic options, ${ }^{45}$ and improvements in its prognostic capability are needed.

New directions include ${ }^{45-51}$ genomics and proteomics of renal disease. This arena is currently handicapped by the need for tissue fixation that may limit recovery for RNA and protein analysis. In addition, the complex structural organization of the kidney with diseases limited to specific segments of the nephron makes interpretation of whole kidney biopsy proteomic or genomic patterns problematic. The latter makes the genomic or proteomic study of glomerulonephritis or tubulointerstitial disease much more difficult than the evaluation of, for example, neoplasms that are far more uniform and often available in much greater quantity. Lasercapture microdissection provides part of the solution by allowing selection of glomeruli or a specific tubular segment for study. ${ }^{52-54}$ Still, the minute amount of available tissue severely limits exploration and further technological advance is needed to validate clinical utility prior to routine application of these exciting techniques.

Conversely, ISH has been successfully applied to various renal diseases during the last 10-15 years. ${ }^{55-59}$ As the availability of reagents and tools has expanded, this technique is being applied more widely. In the future, ISH will provide confirmatory and specific information for the extensive data being generated by genomic studies.

\section{Conclusions}

This document incorporates the consensus opinions of the Ad Hoc Committee on Practice Guidelines, appointed by the Renal Pathology Society. A significant percentage of the membership of the Renal Pathology Society contributed to the manuscript (see Acknowledgements) and the manuscript has received almost universal support from the membership at large. It is intended to highlight the essential ingredients necessary to provide quality renal pathology diagnoses.

Optimum evaluation of a renal biopsy requires a technically proficient laboratory that can generate well-fixed and thinly sectioned slides stained with a panel of special stains. The laboratory must be able to provide complete immunohistochemical analysis 
and ultrastructural examination. Any deviation from this comprehensive array of studies risks incorrect or incomplete diagnostic information.

There is currently no subspecialty certification by the American Board of Pathology for renal pathologists. However, to provide quality diagnoses in this arena, the pathologist should have received more than the usual brief experience in renal pathology during residency. A renal pathology fellowship or a concentrated exposure to renal pathology under the auspices of an experienced nephropathologist is highly recommended.

Since every year new entities are identified and new classification schema are formulated, the provider of renal pathology diagnoses must keep pace with this rapidly evolving subspecialty. This is challenging because the majority of renal pathology contributions to the literature appear in nephrology rather than pathology journals. Furthermore, although the Renal Pathology Society has excellent sponsored programs at the United States and Canadian Academy of Pathology Meeting and other Pathology meetings worldwide, the most extensive renal pathology presentations occur at Nephrology meetings, particularly the American Society of Nephrology Meeting. Thus, significant extra time, effort and expense are required of those desiring to remain current in their knowledge of renal disease.

Renal pathology is an exciting and challenging discipline. Patients with renal disease encompass a broad spectrum of medical diseases that are frequently complex and multisystem in nature. The renal biopsy, appropriately processed and interpreted will yield the correct clinicopathologic diagnosis leading to the appropriate therapeutic strategy while, at the same time, providing key prognostic information.

\section{Acknowledgements}

The members of the Renal Pathology Society who contributed to the development of this article include (in alphabetical order):

Arend LJ, Benediktsson H, Bonsib SM, Brisson M-L, Bruijn JA, Cavallo T, Chander PN, Charney DA, Cohen AH, Colvin RB, Crary GS, D’Agati V, Fogo AB, Ferrario F, Gokden N, Haas M, Harris A, Herrera GA, Iskander S, Jennette C, Kashgarian M, King JA, Liapis H, Markowitz G, Meleg-Smith S, Mengel M, Mölne J, Moura LA, Nair R, Nasr H, Nast CC, Neff JC, Nickeleit V, Noel L-H, Olsen S, Phillips C, Racusen L, Reinholt FP, Rosen S, Ruiz P, Salinas-Madrigal L, Sarioglu S, Schwartz MM, Sens MA, Seshan SV, Solez K, Strom EH, Truong LD, Walker PD, Youngberg GA, Zhou XJ.

\section{Duality of interest}

None declared.

\section{References}

1 Khajehdehi P, Junaid SMA, Salinas-Madrigal L, et al. Percutaneous renal biopsy in the 1990s: safety, value, and implications for early hospital discharge. Am J Kidney Dis 1999;34:92-97.

2 Hefter LG, Brennan GG. Transillumination of renal biopsy specimens for rapid identification of glomeruli. Kidney Int 1981;20:411-415.

3 Sheehan DC, Hrapchak BB. Theory and Practice of Histotechnology. Battelle Press: Columbus, OH: 1980.

4 Lajoie G, Silva FG. Technical aspects of renal biopsy processing. In: Silva FG, D’Agati VD, Nadasdy T (eds). Renal Biopsy Interpretation. Churchill Livingstone: New York, 1996, pp 423-435.

5 Matsuno A, Nagashima T, Ohsugi Y, et al. Electron microscopic observation of intracellular expression of mRNA and its protein product: technical review on ultrastructural in situ hybridization and its combination with immunohistochemistry. Histol Histopathol 2000;15:261-268.

6 Terenghi G. Preparation of tissue sections and slides for mRNA hybridization. Methods Mol Biol 1998; 86:133-136.

7 Shibutani M, Uneyama C. Methacarn: a fixation tool for multipurpose genetic analysis from paraffin-embedded tissues. Methods Enzymol 2002;356:114-125.

8 Scheidl SJ, Nilsson S, Kalen M, et al. mRNA expression profiling of laser microbeam microdissected cells from slender embryonic structures. Am J Pathol 2002; 160:801-813.

9 Wang NS, Minassian H. The formaldehyde-fixed and paraffin-embedded tissues for diagnostic transmission electron microscopy: a retrospective and prospective study. Hum Pathol 1987;18:715-727.

10 Widehn S, Kindblom LG. A rapid and simple method for electron microscopy of paraffin-embedded tissue. Ultrastruct Pathol 1988;12:131-136.

11 van den Berg Weerman MA, Dingemans KP. Rapid deparaffinization for electron microscopy. Ultrastruct Pathol 1984;7:55-57.

12 Mac-Moune Lai F, Lai KN, Chew EC, et al. Microwave fixation in diagnostic renal pathology. Pathology 1987; 19:17-21.

13 Rohr LR, Layfield LJ, Wallin D, et al. A comparison of routine and rapid microwave tissue processing in a surgical pathology laboratory. Quality of histologic sections and advantages of microwave processing. Am J Clin Pathol 2001;115:703-708.

14 Leong AS. Microwave fixation and rapid processing in a large throughput histopathology laboratory. Pathology 1991;23:271-273.

15 Michel B, Milner Y, David K. Preservation of tissuefixed immunoglobulins in skin biopsies of patients with lupus erythematosus and bulous diseasespreliminary report. J Invest Dermatol 1972;59:449-452.

16 Strege RJ, Saeger W, Linke RP. Diagnosis and immunohistochemical classification of systemic amyloidoses: report of 43 cases in an unselected autopsy series. Virchows Arch 1998;433:19-27.

17 Lofberg H, Thysell H, Westman K, et al. Demonstration and classification of amyloidosis in needle biopsies of the kidneys, with special reference to amyloidosis of the AA-type. Acta Pathol Microbiol Immunol Scand A 1987;95:357-363.

18 Pirson Y. Making the diagnosis of Alport's syndrome. Kidney Int 1999;56:760-775. 
19 Kashtan CE. Alport syndrome and thin glomerular basement membrane disease. J Am Soc Nephrol 1998;9:1736-1750.

20 Fakhouri F, Darre S, Droz D, et al. Mesangial IgG glomerulonephritis: a distinct type of primary glomerulonephritis. J Am Soc Nephrol 2002;13:379-387.

21 Zuniga R, Markowitz GS, Arkachaisri T, et al. Identification of IgA subclasses and C-reactive protein in lupus nephritis: the relationship between the composition of immune deposits an FC gamma receptor type IIA alleles. Arthritis Rheum 2003;48: 460-470.

22 Nickeleit V, Hirsch HH, Binet IF, et al. Polyomavirus infection of renal allograft recipients: from latent infection to manifest disease. J Am Soc Nephrol 1999;10:1080-1089.

23 Detwiler RK, Singh HK, Bolin P, et al. Cytomegalovirus-induced necrotizing and crescentic glomerulonephritis in a renal transplant patient. Am J Kidney Dis 1998;32:820-824.

24 Asim M, Chong-Lopez A, Nickeleit V. Adenovirus infection of a renal allograft. Am J Kidney Dis 2003; 41:696-701.

25 Szymanski IO, Pullman JM, Underwood JM. Electron microscopic and immunochemical studies in a patient with hepatitis $\mathrm{C}$ virus infection and mixed cryoglobulinemia type II. Am J Clin Pathol 1994;102:278-283.

26 Trpkov K, Marcussen N, Rayner D, et al. Kidney allograft with a lymphocytic infiltrate: acute rejection, posttransplantation lymphoproliferative disorder, neither, or both entities. Am J Kidney Dis 1997;30: 449-454.

27 Meehan SM, Domer P, Josephson M, et al. The clinical and pathologic implications of plasmacytic infiltrates in percutaneous renal allograft biopsies. Hum Pathol 2001;32:205-215.

28 Nickeleit V, Zeiler M, Gudat F, et al. Detection of the complement degradation product C4d in renal allografts: diagnostic and therapeutic implications. J Am Soc Nephrol 2002;13:242-251.

29 Mauiyyedi S, Crespo M, Collins AB, et al. Acute humoral rejection in kidney transplantation: II. Morphology, immunopathology, and pathologic classification. J Am Soc Nephrol 2002;13:779-787.

30 Pirani CL, Croker BP. Handling and processing of renal biopsy and nephrectomy specimens. In: Tisher CC, Brenner BM (eds). Renal Pathology. J.B. Lippincott Co.: Philadelphia, PA, 1994, pp 1683-1694.

31 Schwartz MM. The pathologic diagnosis of renal disease. In: Jennette JC, Olson JL, Schwartz MM, Silva FG (eds). Heptinstall's Pathology of the Kidney. Lippincott-Raven Publications: Philadelphia, PA, 1998, pp 169-180.

32 Rivera A, Meleg-Smith S. Value of electron microscopy in the diagnosis of childhood nephrotic syndrome. Ultrastruct Pathol 2001;25:313-320.

33 Furness PN. Renal biopsy specimens. J Clin Pathol 2000;53:433-438.

34 Haas M. A reevaluation of routine electron microscopy in the examination of native renal biopsies. J Am Soc Nephrol 1997;8:70-76.

35 Randhawa PS, Minervini MI, Lombardero M, et al. Biopsy of marginal donor kidneys: correlation of histologic findings with graft dysfunction. Transplantation 2000;69:1352-1357.

36 Escofet X, Osman H, Griffiths DF, et al. The presence of glomerular sclerosis at time zero has a significant impact on function after cadaveric renal transplantation. Transplantation 2003;75:344-346.

37 Wang HJ, Kjellstrand CM, Cockfield SM, et al. On the influence of sample size on the prognostic accuracy and reproducibility of renal transplant biopsy. Nephrol Dial Transplant 1998;13:165-172.

38 Herzenberg AM, Gill JS, Djurdjev O, et al. C4d deposition in acute rejection: an independent longterm prognostic factor. J Am Soc Nephrol 2002;13: 234-241.

39 Colvin RB, Cohen AH, Saiontz C, et al. Evaluation of pathologic criteria for acute renal allograft rejection: reproducibility, sensitivity, and clinical correlation. J Am Soc Nephrol 1997;8:1930-1941.

40 Solez K, Axelsen RA, Benediktsson H, et al. International standardization of criteria for the histologic diagnosis of renal allograft rejection: the Banff working classification of kidney transplant pathology. Kidney Int 1993;44:411-422.

41 Racusen LC, Solez K, Colvin RB, et al. The Banff 97 working classification of renal allograft pathology. Kidney Int 1999;55:713-723.

42 Racusen LC, Colvin RB, Solez K, et al. Antibodymediated rejection criteria - an addition to the Banff '97 classification of renal allograft rejection. Am J Transplant 2003;3:1-7.

43 Cotran RS. Fifth annual John Peters Award, American Society of Nephrology. Award recipients: Jacob Churg and Conrad Pirani. Kidney Int 1988;34:121-123.

44 Pirani CL. Renal biopsy: an historical perspective. In: Silva FG, D’Agati VD, Nadasdy T (eds). Renal Biopsy Interpretation. Churchill Livingstone: New York, 1996, pp 1-19.

45 Kretzler M, Cohen CD, Doran P, et al. Repuncturing the renal biopsy: strategies for molecular diagnosis in nephrology. J Am Soc Nephrol 2002;13:1961-1972.

46 Eikmans M, Baelde HJ, Hagen EC, et al. Renal mRNA levels as prognostic tools in kidney diseases. J Am Soc Nephrol 2003;14:899-907.

47 Eikmans M, Baelde HJ, de Heer E, et al. RNA expression profiling as prognostic tool in renal patients: toward nephrogenomics. Kidney Int 2002;62: 1125-1135.

48 Arthur JM, Thongboonkerd V, Scherzer JA, et al. Differential expression of proteins in renal cortex and medulla: a proteomic approach. Kidney Int 2002;62: 1314-1321.

49 Striker LJ, Striker GE. Windows on renal biopsy interpretation: does mRNA analysis represent a new gold standard? J Am Soc Nephrol 2003;14:1096-1098.

50 Knepper MA. Proteomics and the kidney. J Am Soc Nephrol 2002;13:1398-1408.

51 Kurella M, Hsiao L, Yoshida T, et al. DNA microarray analysis of complex biologic processes. J Am Soc Nephrol 2001;12:1072-1078.

52 Cohen CD, Grone H, Grone EF, et al. Laser microdissection and gene expression analysis on formaldehyde-fixed archival tissue. Kidney Int 2002;61: 125-132.

53 Gjerdrum LM, Lielpetere I, Rasmussen LM, et al. Laser-assisted microdissection of membrane-mounted paraffin sections for polymerase chain reactions analysis. J Mol Diagn 2001;3:105-110.

54 Nagasawa Y, Takenaka M, Matsuoka Y, et al. Quantitative of mRNA expression in glomeruli using lasermanipulated microdissection and laser pressure catapulting. Kidney Int 2000;57:717-723. 
55 Payton D, Thorner P, Eddy A, et al. Demonstration by light microscopy of cytomegalovirus on a renal biopsy of a renal allograft recipient: confirmation by immunohistochemistry and in situ hybridization. Nephron 1987;47:205-208.

56 Rastaldi MP, Ferrario F, Giardino L, et al. Epithelialmesenchymal transition of tubular epithelial cells in human renal biopsies. Kidney Int 2002;62:137-146.

57 Schaefer L, Grone H-J, Raslik I, et al. Small proteoglycans of normal adult human kidney: distinct expres- sion patterns of decorin, biglycan, fibromodulin, and lumican. Kidney Int 2000;58:1557-1568.

58 Segerer S, Cui Y, Eitner F, et al. Expression of chemokines and chemokine receptors during human renal transplant rejection. Am J Kidney Dis 2001;37: $518-531$.

59 Anders H-J, Vielhauer V, Kretzler M, et al. Chemokine and chemokine receptor expression during initiation and resolution of immune complex glomerulonephritis. J Am Soc Nephrol 2001;12:919-931. 